Supporting information for:

\title{
Nucleating Pattern Formation in Spin-Coated Polymer Blend Films with Nanoscale Surface Templates
}

Joseph H. Wei ${ }^{1}$; David C. Coffey ${ }^{2}$; David S. Ginger ${ }^{3}$

1. Department of Chemical Engineering, University of Washington, Seattle, WA 98195-1750

2. Department of Physics, University of Washington, Seattle, WA 98195-1560

3. Department of Chemistry, University of Washington, Seattle, WA 98195-1700

\section{Experimental Details}

The XPS has a monochromatized Al X-ray and a low energy electron flood gun for charge neutralization. An X-ray spot size for these acquisitions was $800 \mu \mathrm{m}$ by $800 \mu \mathrm{m}$. The take-off angle (the angle between the sample normal and the input axis of the energy analyzer) was $\sim 55^{\circ}$ ( $55^{\circ}$ take-off angle @ $50 \AA$ A sampling depth). Pressure in the analytical chamber during spectral acquisition was less than $5 \times 10^{-9}$ Torr. The pass energy for survey spectra (composition) was $150 \mathrm{eV}$. The Service Physics ESCA Analysis 2000 program was used to determine peak areas and to calculate the elemental compositions from peak areas. The binding energy scale was calibrated by assigning the $\mathrm{C} 1 \mathrm{~s}$ peak to a binding energy of $285.0 \mathrm{eV}$. 
Supplementary Figures
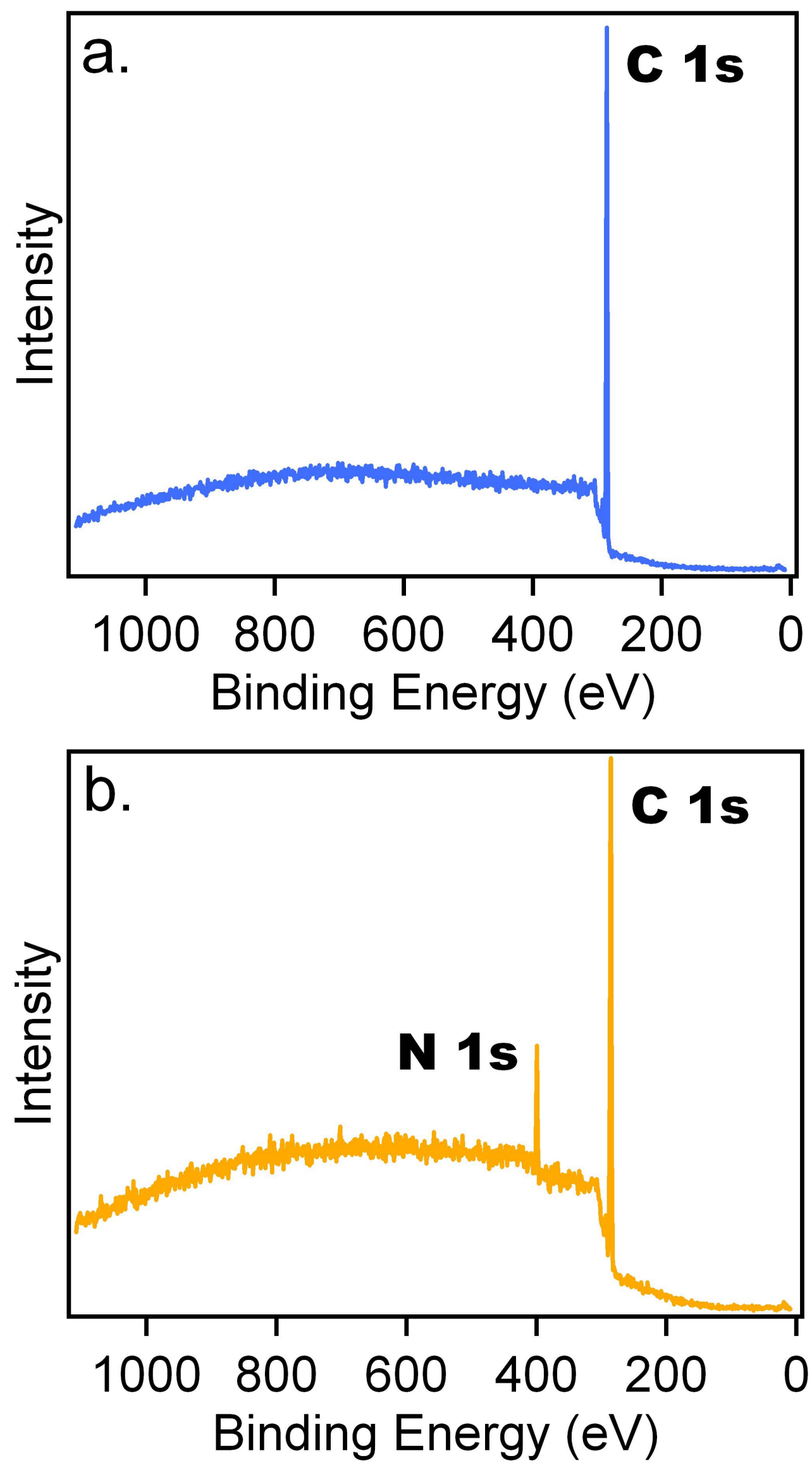


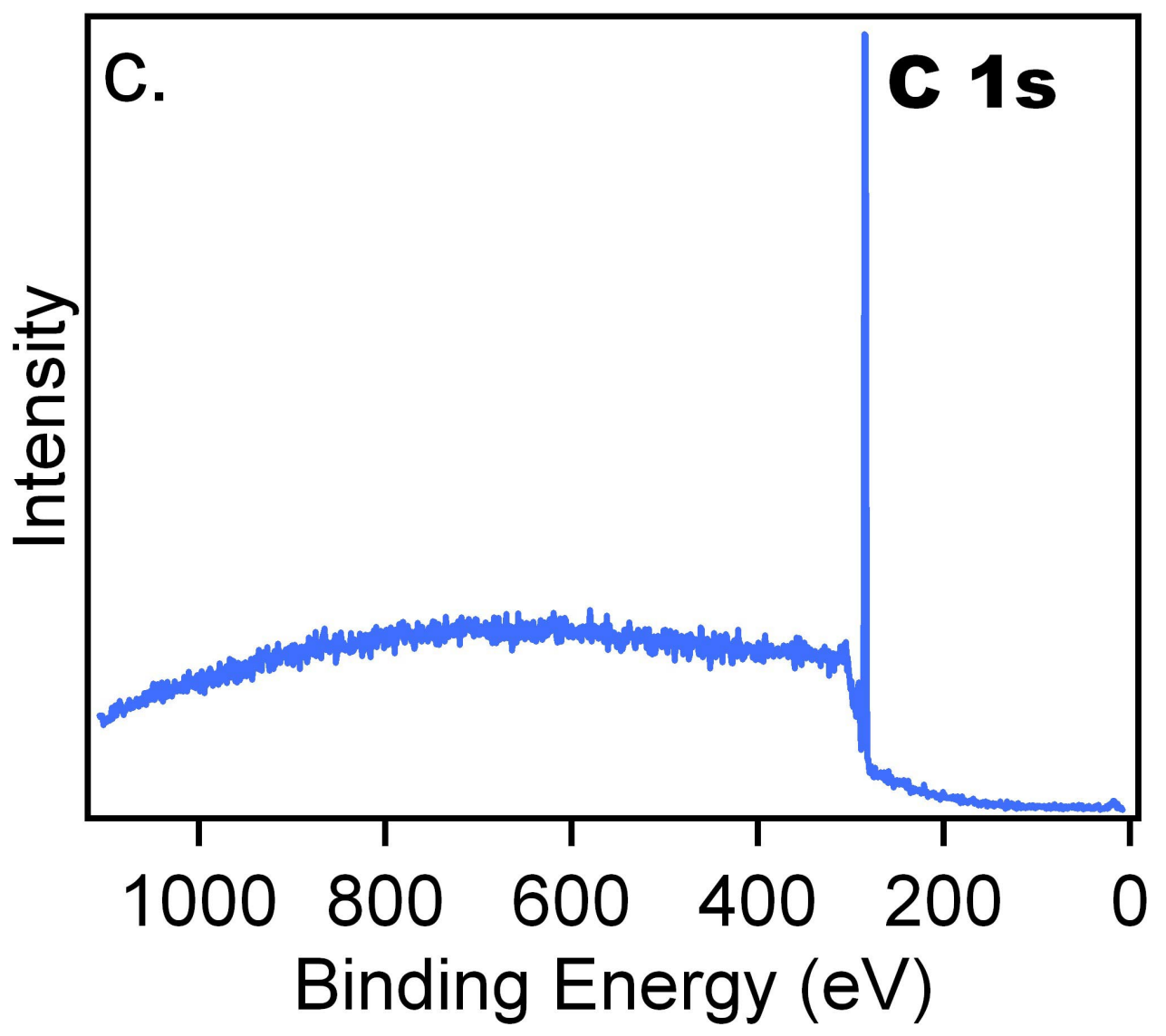

Figure S1. XPS spectra of a) PS surface, b) P2VP surface, and c) 50/50 PS/P2VP blend film (unpatterned).

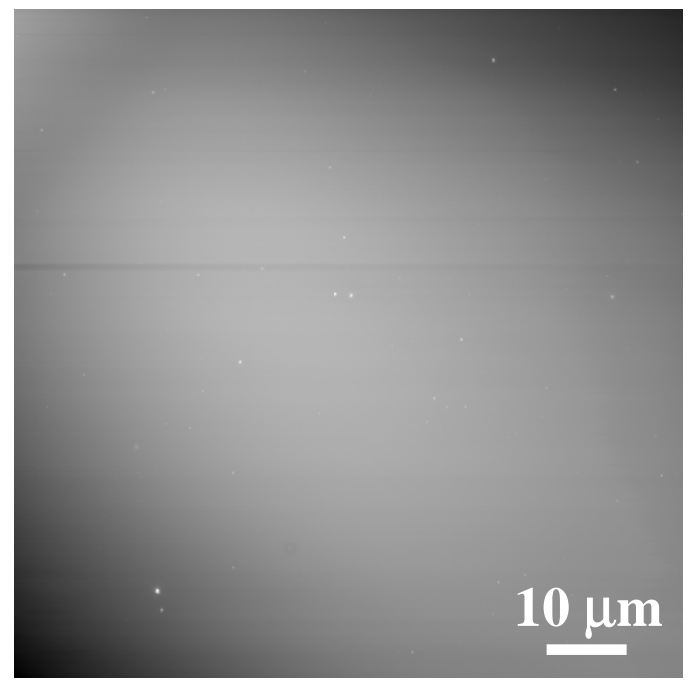

Figure S2. AFM topography image of a PS/P2VP (50/50) blend spin-coated onto a DPN-generated MHA template with benzenethiol background on gold. 

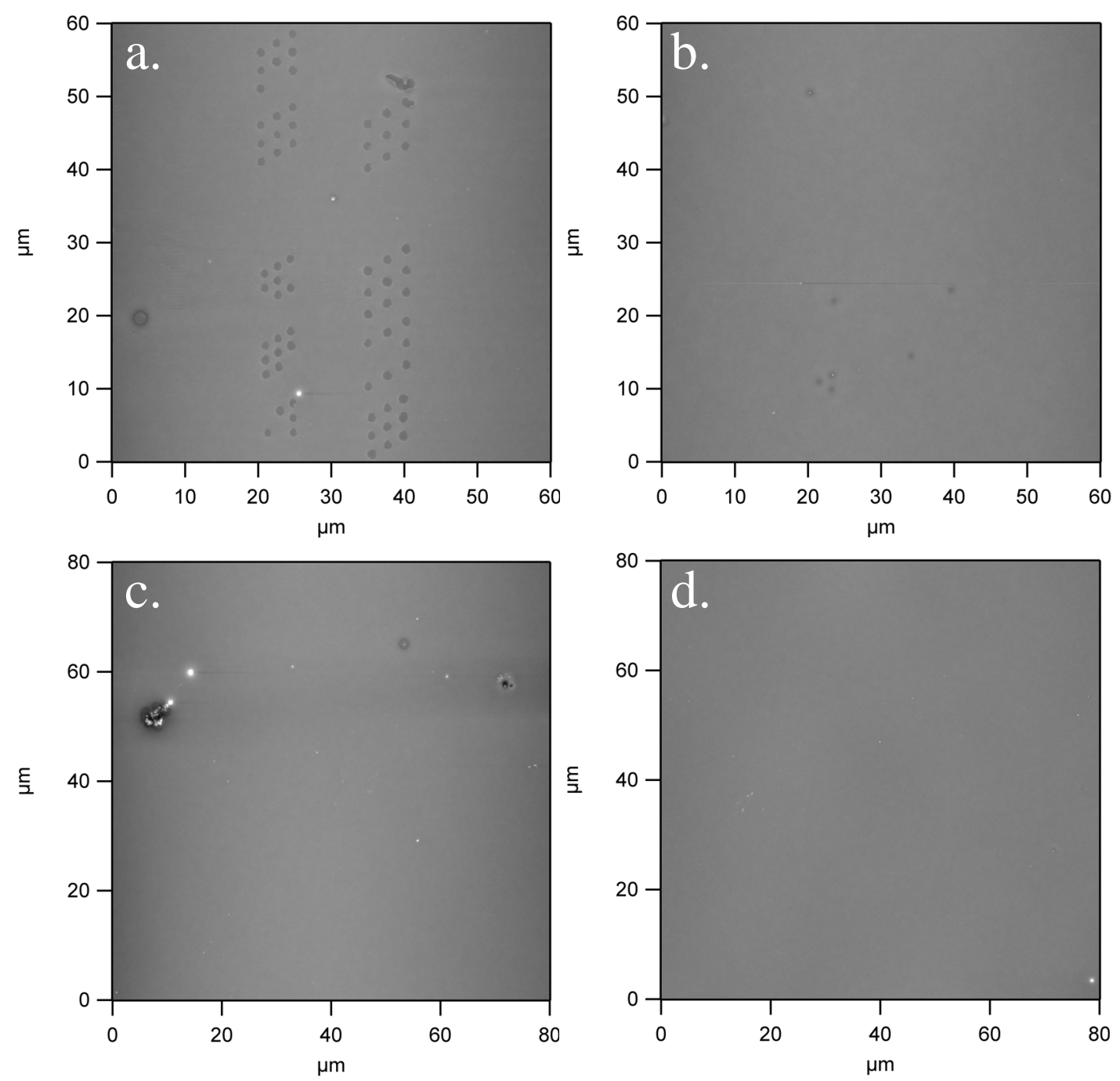

Figure S3. Comparisons of Pattern Formation in PS/P2VP Blends with DPN-Generated ODT Templates in Various Background Chemistry. AFM topography images of PS/P2VP (50/50) blends spin-coated onto ODT templates with a) benzenethiol background on gold, b) 6-amino-1hexanethiol(hydrochloride salt) background on gold, c) MHA background on gold, and d) 11mercapto-1-undecanol background on gold (z-range: $50 \mathrm{~nm}$ ). 

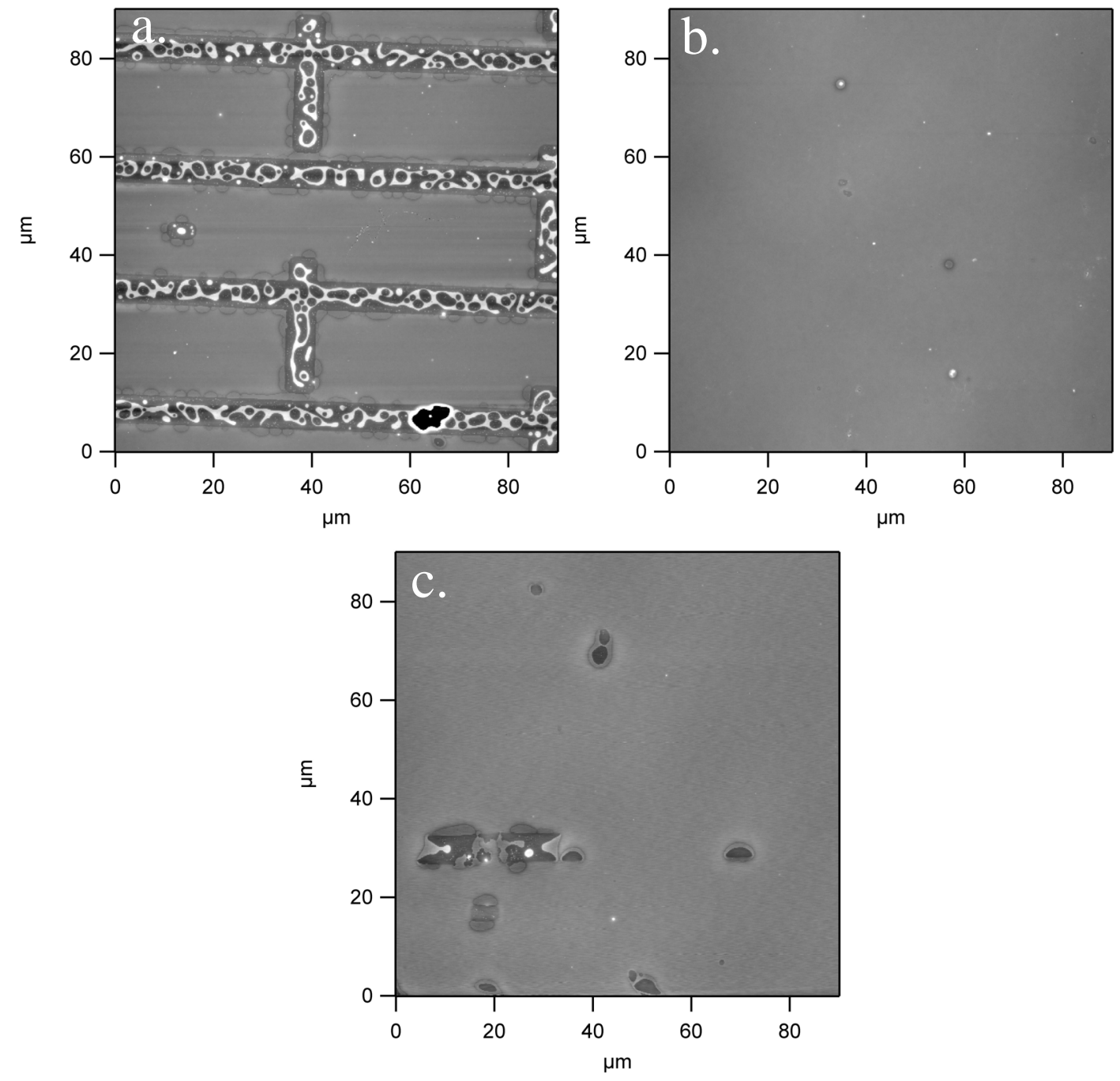

Figure S4. Comparisons of Pattern Formation in PS/P2VP Blends with Micro Contact-Printed ODT Templates in Various Background Chemistry. AFM topography images of PS/P2VP (50/50) blends spin-coated onto ODT templates with a) benzenethiol background on gold, b) MHA background on gold, and c) 11-mercapto-1-undecanol background on gold (z-range: $50 \mathrm{~nm}$ ). 

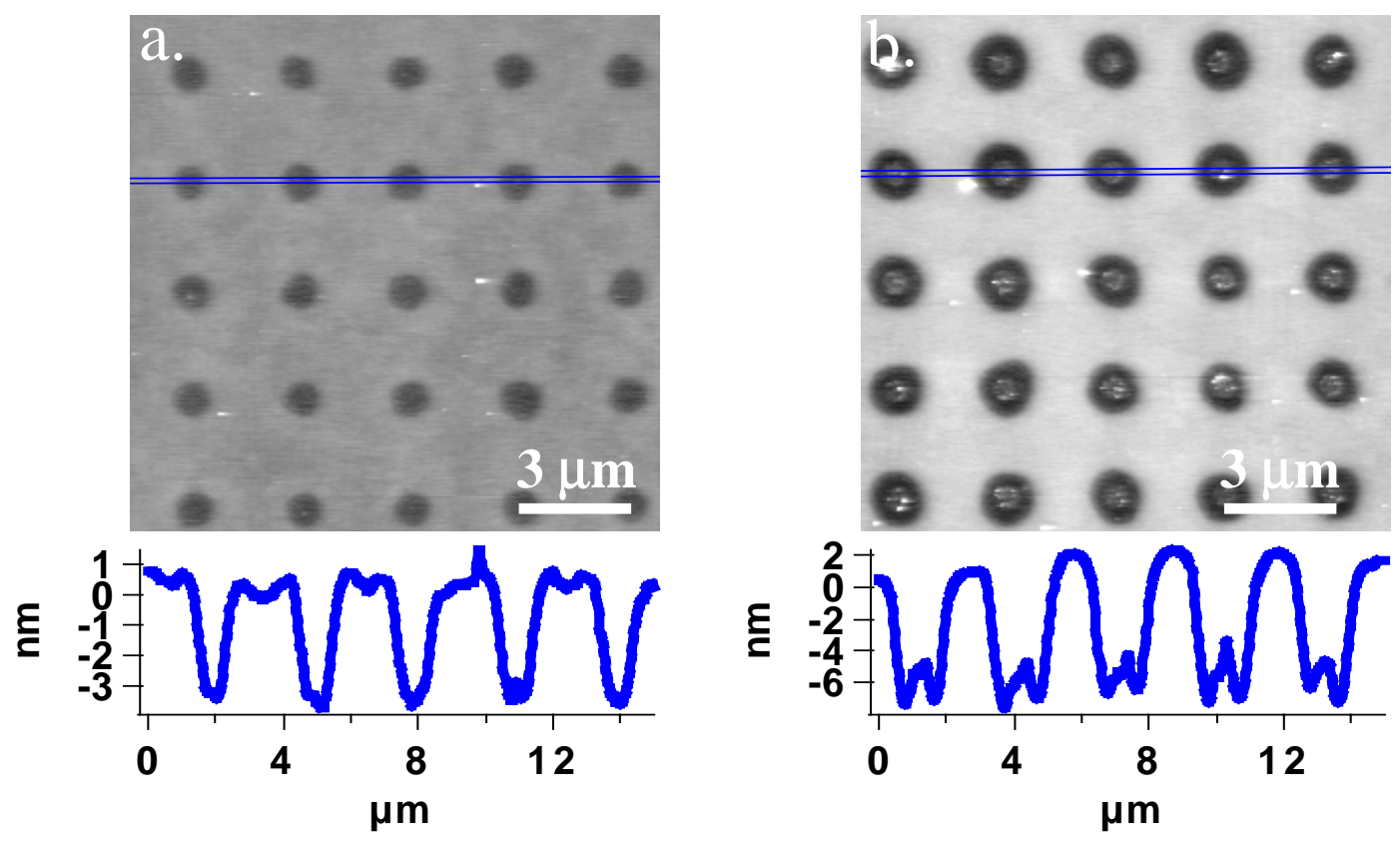

Figure S5. Comparisons of Pattern Formation in PS/P3HT Blends with MHA and ODT Templates (Dot Diameter: 500 nm). AFM topography images of PS/P3HT (60/40) blends spincoated onto a) a DPN-generated MHA template with benzenethiol background on gold and b) a DPNgenerated ODT template with benzenethiol background on gold (z-range: $10 \mathrm{~nm}$ ).

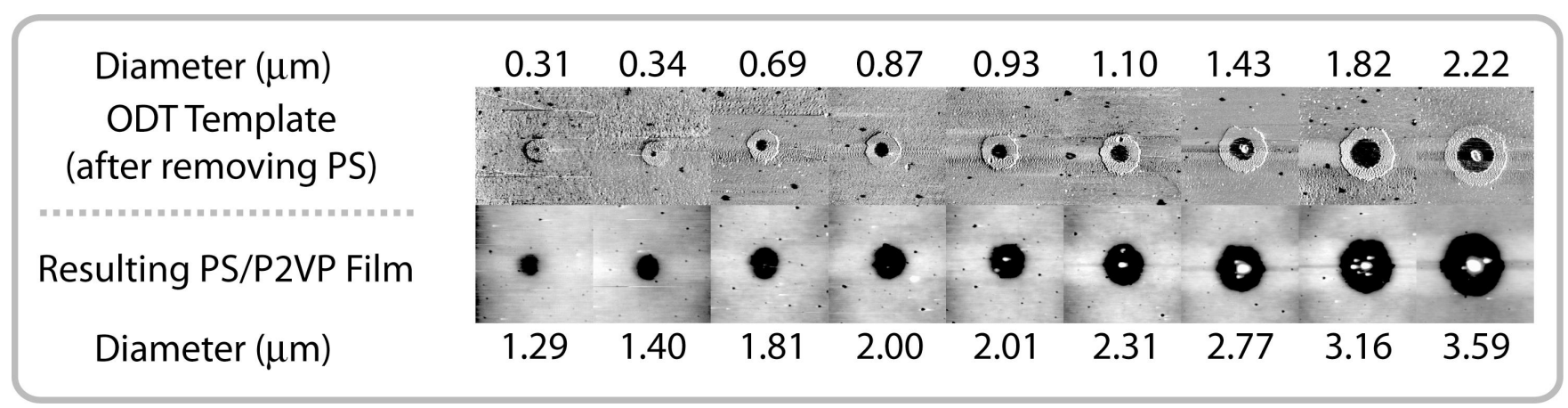

Figure S6. Evolution of Film Morphology with Template Size for PS/P2VP Blends without Observation of the Rim Feature. Lateral force images (top: after cyclohexane etching) and AFM topography (bottom: before cyclohexane etching) of a 50/50 PS/P2VP blend on ODT templates of various diameters. 


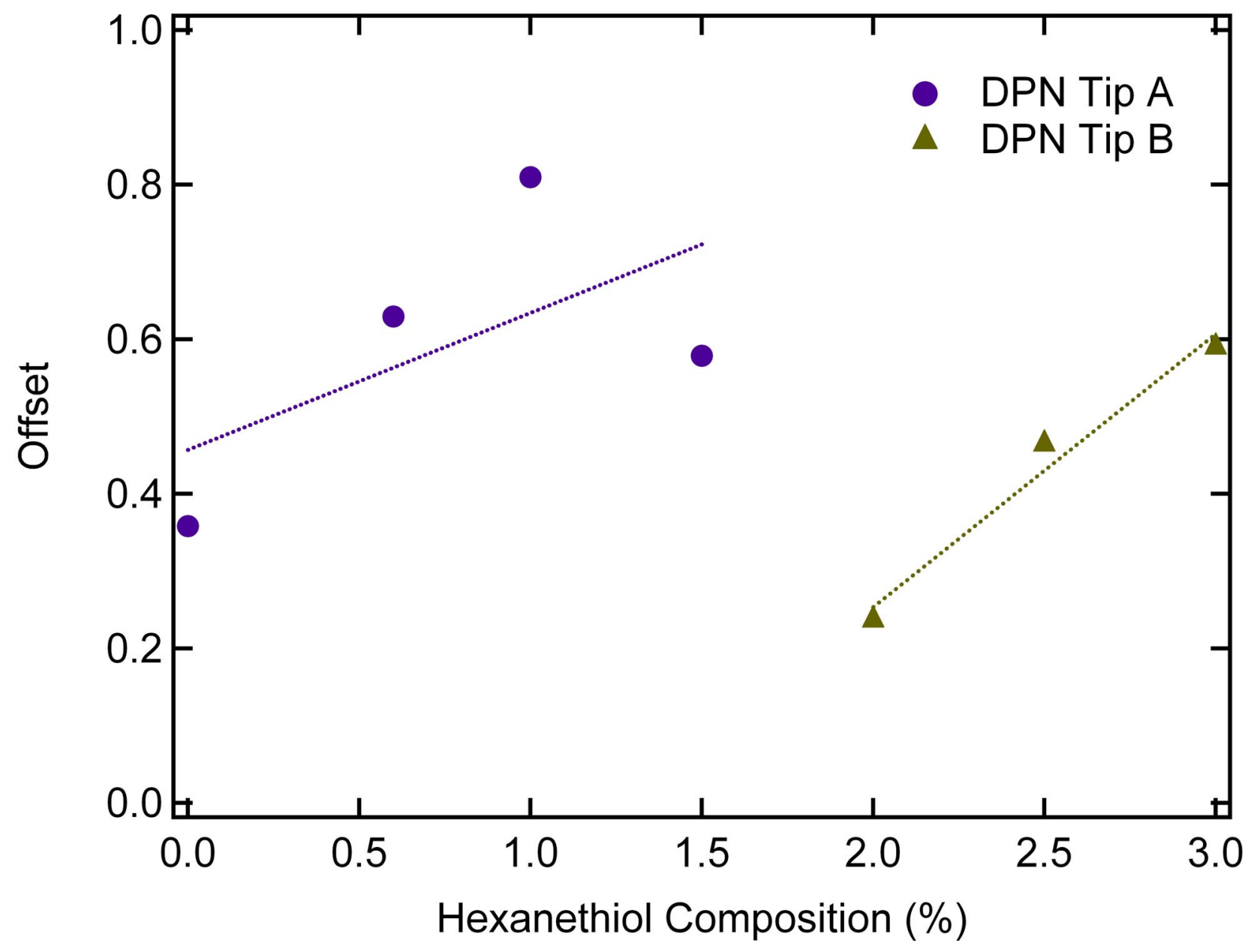

Figure S7. Offsets from the Polymer Domain Diameter vs. Template Diameter Plot (e.g. Fig. 6a in the Main Text) as a Function of the Hexanethiol Composition of the Backfill. This control study supports a possible hypothesis as to why the intercepts/offsets (corresponding to the distance the hole has grown past the edge of the template) in Fig. 6a vary from sample to sample: different degrees of wettability in the background around the templates from inadvertent contamination. We intentionally introduce hexanethiol as contamination in benzenethiol for background passivation. Fig. S4 indicates that in general backfilling with higher concentrations of hexanethiol led to faster dewetting and larger "offsets". It is expected to see different trends with different DPN tips because of the variations in the amount of ODT vapor transport overtime. The lines are least-square fits to the data points. 


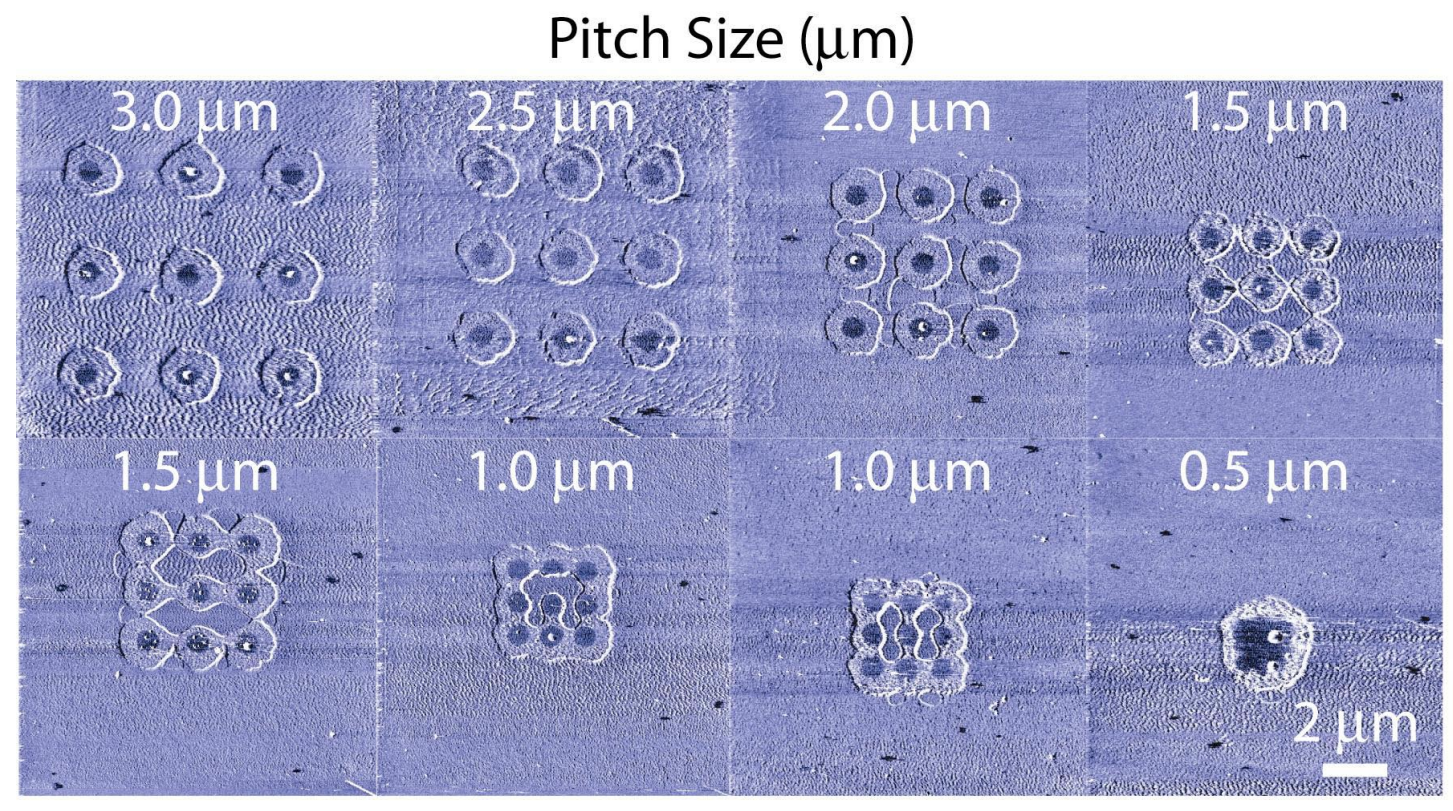

Figure S8. Effect of Template Pitch on Polymer Feature Size. Lateral force (friction) images of $\mathrm{PS} / \mathrm{P} 2 \mathrm{VP}$ blends (50/50) showing that changes in the polymer feature diameter as the template pitch size decreases are smaller in the PS/P2VP system than the PS/P3HT system up to the point the pattern formations begin to merge.

\section{Supplementary Tables}

Table S1. Selective Etching Study of PS/PVP Films. Cyclohexane is used to selectively etch the PS films, but not the PVP films, to verify the hypothesized vertical bilayer structure. Total film thickness before and after etching in different blends of PS/P2VP is measured with a scanning probe microscope.

\begin{tabular}{|c|c|c|c|c|c|}
\hline PS/P2VP & $\begin{array}{c}\text { Film thickness } \\
\text { before cyclohexane } \\
\text { etching (nm) }\end{array}$ & $\begin{array}{c}\text { Film thickness after } \\
\text { cyclohexane etching } \\
\text { (nm) }\end{array}$ & $\begin{array}{c}\text { Percentage changed } \\
\text { before and after } \\
\text { etching (\%) }\end{array}$ & $\begin{array}{c}\text { Deduced PS film } \\
\text { thickness (nm) }\end{array}$ & $\begin{array}{c}\text { Deduced P2VP film } \\
\text { thickness (nm) }\end{array}$ \\
\hline \hline$(50 / 50)$ & 31 & 16 & 52 & 15 & 16 \\
\hline$(33 / 67)$ & 27 & 15 & 56 & 12 & 15 \\
\hline$(25 / 75)$ & 30 & 23 & 77 & 7 & 23 \\
\hline$(20 / 80)$ & 28 & 25 & 89 & 3 & 25 \\
\hline$(17 / 83)$ & 26 & 22 & 85 & 4 & 22 \\
\hline
\end{tabular}

